\title{
"War, Migration and Health: What Should Physicians Do»
}

\section{Christine Romann}

Dr med., membre du Comité central de la FMH, responsable du département Promotion de la santé et prévention

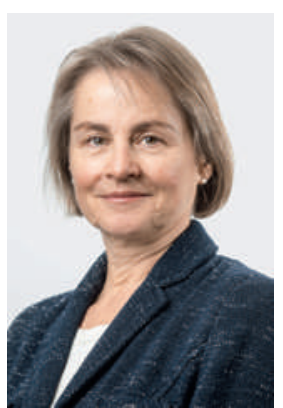

Conjointement avec plusieurs organisations médicales turques, l'Association médicale mondiale (AMM) a organisé fin février dernier à Istanbul un symposium sur un sujet brûlant de l'actualité: «War, Migration and Health: What Should Physicians Do». Les représentants de plusieurs organisations médicales nationales ont évoqué la situation dans leur pays, notamment les problèmes et les solutions proposées pour la prise en charge médicale des migrants. Différents exposés ont ensuite permis de mieux se rendre compte de la situation actuelle des réfugiés. Un représentant de Médecins sans frontières (MSF) a souligné le travail de son organisation, gage de survie pour des milliers de réfugiés. Dans des conditions souvent très difficiles, les médecins de MSF garantissent la prise en charge médicale de base dont les migrants ont urgemment besoin, parfois dans des circonstances révoltantes et au péril de leur vie. Au Yémen par exemple, leurs hôpitaux, qui sont la seule possibilité de dispenser des soins, sont la cible de bombardements de la part des forces armées saoudiennes. En Syrie, le régime de Bachar al-Assad bombarde également les hôpitaux; des médecins et des infirmiers ont même été assassinés parce qu'ils soignaient tous les patients, indépendamment de leur appartenance politique. Ce qui devrait être indissociable de la déontologie et de l'action médicale devient un danger mortel.

\section{Un développement global et durable, offrant} à tous un meilleur environnement économique et davantage de sécurité, est nécessaire.

Ce n'est pas un hasard si Istanbul a été choisie pour accueillir ce symposium. En effet, la Turquie constitue la première étape pour de nombreux réfugiés. Au fil des interventions, chacun a pu se rendre compte des enjeux dramatiques que représente l'accueil d'environ trois millions de personnes dans un pays dont la population avoisine celle de l'Allemagne. Seuls dix pour cent des migrants vivent dans les 25 camps de réfugiés prévus le long de la frontière syrienne. Les autres, soit près de deux millions et demi de personnes, sont dispersés dans les villes ou attendent sur les bords de la mer Egée de pouvoir se lancer dans la périlleuse traversée vers
l'Europe; tous vivent dans des conditions très précaires. Lors du symposium, l'AMM, les organisations médicales turques et étrangères présentes ont approuvé un document de travail ${ }^{1}$ qui servira de base à l'AMM pour la poursuite de son engagement. Ce document souligne entre autres que les médecins savent l'attention qu'il faut porter aux déterminants de la santé et qu'ils s'engagent en faveur de conditions de vie acceptables pour toutes les personnes, qu'elles soient en fuite ou acceptées dans un pays d'accueil. Aucune différence ne doit être faite entre celles qui ont quitté leur terre d'origine pour des raisons de guerre, de violence ou de répression

\section{Les personnes en fuite ont besoin de protec-} tion, de nourriture, d'eau salubre et de bons soins médicaux.

politique, et celles qui veulent fuir la pauvreté, le manque de perspective ou la faim: toutes aspirent à des conditions de vie meilleures et plus sûres, pour elles et pour leur famille. Le document rappelle aussi que seul un développement global et durable, offrant à tous un meilleur environnement économique et davantage de sécurité, pourra stopper le flux de réfugiés.

Au terme du symposium, trois maires turcs ont fait part de leurs expériences dans leur commune respective. Un petit film en a dit bien davantage que tous les mots: dans «Sorry I drowned» (à consulter sur Youtube en indiquant ce titre), un jeune syrien rend hommage à son ami noyé. Pendant ce temps, des photos de la plage d'une petite ville circulaient parmi le public: des corps sans vie rejetés par la mer. Une jeune femme, un vieil homme et deux personnes en pleine force de l'âge sortent de la plage un petit corps enroulé dans une couverture de survie, leurs visages reflètent toute la catastrophe.

Que peuvent faire les médecins? Avec ce document, l'AMM définit un axe de réflexion et marque son soutien: les médecins peuvent et doivent rappeler qu'il ne s'agit pas simplement d'un flux menaçant de réfugiés vers l'Europe mais de personnes à la recherche d'un lieu sûr pour vivre: des femmes, des enfants, des hommes. Ils ont droit à la dignité, à notre protection, ils ont besoin de nourriture, d'eau salubre et de bons soins médicaux. 